\title{
Porous and Nonporous Film-Shaped Magnetorheological Nanocomposites: Dielectric and Electrical Properties
}

\author{
Aref Naimzad, Yousef Hojjat, and Mojtaba Ghodsi \\ Manufacturing Department, Mechanical Engineering Faculty, Tarbiat Modares University, Tehran 1411713116, Iran \\ Correspondence should be addressed to Aref Naimzad; naimzad@modares.ac.ir
}

Received 2 July 2014; Revised 18 September 2014; Accepted 4 November 2014; Published 1 December 2014

Academic Editor: Osama J. Aldraihem

Copyright (c) 2014 Aref Naimzad et al. This is an open access article distributed under the Creative Commons Attribution License, which permits unrestricted use, distribution, and reproduction in any medium, provided the original work is properly cited.

\begin{abstract}
This paper presents a brief experimental comparative study on electrical and dielectric properties of two sets of porous and nonporous MRNCs, each including five samples of film-shaped magnetorheological nanocomposites (MRNCs) based on room temperature vulcanized (RTV) silicone rubber and nanosized carbonyl iron particles (CIPs). The electrical and dielectric properties of porous and nonporous MRNCs were measured at five different filler concentrations. Several experiments were performed to measure the volume resistivity, dielectric constant, and dielectric loss. The MRNCs dielectric properties were analysed with respect to the parameters like frequency and CIPs loadings. The electrical conductivity was studied in terms of volume resistivity. The comparative investigation suggests the porous MRNCs for smart and light-weighted structures those benefits from a lower electrical property, dielectric losses, and dielectric constants.
\end{abstract}

\section{Introduction}

Magnetorheological nanocomposites (MRNCs) are the new category of smart materials which are sensitive against applied magnetic fields. MRNCs consisting of nanosized CIPs embedded in a silicone rubber matrix display a variety of interesting properties to design the flexible actuators [1-5].

The authors' previous works [3-5] were focused on fabricating a light-weight and flexible nonporous MRNCs for miniature gripper applications using laser ablated nanopowder of CIPs. The magnetic and mechanical property values of manufactured MRNCs were determined and the different fabrication technologies have been tested as hot press, chemical vacuum vaporization, and laser beam moulding to study the property changes which benefit the operational condition of miniature actuators.

The authors of the recent work [6] are concerned to report the manufacturing of porous and nonporous MRNCs and its comparison considering mechanical and magnetic properties variations. The work discussed the fabrication and characterization aspects of porous and nonporous MRNCs in detail.
As the literature indicates there is no reference research work reported directly to the electrical and dielectric properties of MRNCs yet and available published articles are concerning to the part of MR family composites as MR elastomers and silicone rubbers.

According to the literature, enhanced electrical conductivity was observed in the modified multiwalled CNTs/methyl vinyl silicone rubber nanocomposites which is 7-order of magnitude larger than that of silicone rubber host [7].

In silicone rubber composites filled with CIPs, the variation of dielectric constant and loss values depended on the frequency increase and mostly the decreasing trends appeared during the frequency cycle [8]. The electroceramic fillers such as titanium dioxide can increase the dielectric constant and losses of a silicone rubber composite. Also, there will be a considerable boost in the dielectric constant of the oriented composite compared with randomly distributed titania particles [9].

It was examined that the values of relative dielectric constant increase with the increasing frequency and content of titanium diboride $\left(\mathrm{TiB}_{2}\right)$ nanoparticles in a silicone rubber matrix [10] at a frequency range of $1-12 \mathrm{GHz}$. 
The electrical conductivity of the as-prepared CNTs/silicone rubber nanocomposites is increased with the increase of CNTs loading and through further increase in CNTs loading, more conductive paths are formed [11].

In silicone rubber microcomposites based on combination of ferrite and CIPs, the dielectric loss is increased in some desired frequency range of $2-18 \mathrm{GHz}$ [12]. In a comparison work of silicone rubber and epoxy based composites filled with CIPs [13], it has been declared that real permittivity of silicone rubber based composite is lower than epoxy based composites, whilst the imaginary permittivity of silicone rubber based composite is higher than epoxy based composites regarding a frequency range of $26-40 \mathrm{GHz}$. The silicone rubber nanocomposites filled with titanium dioxide and barium titanate synthesized over a frequency range from $100 \mathrm{~Hz}$ to $10 \mathrm{MHz}$ [14] and its findings show that the dielectric permittivity increases whereas dielectric losses decrease with increasing filler concentration.

A mathematical relation for magnetorheological resistance was introduced to measure the electrical resistivity as a function of applied forces on the surface of samples [15] considering the fixed values of transverse magnetic field strength. The work summarized that MREs become electroconductive for volume concentrations of magnetizable phase greater than $40 \%$.

Dependency of the dielectric permittivity on mass fraction of carbon black was reported on silicone rubber/carbon black nanocomposites incorporated with $\mathrm{BaTiO}_{3}[16]$ and claimed that the dielectric loss of the nanocomposites increased with increase of mass fraction of carbon black.

Recently, the measurement of dielectric strength, dielectric constant, volume and surface resistivity of EPDM/silicone rubber nanocomposites incorporated of organically modified montmorillonite (OMMT) nanoclay discussed [17] and concluded that the presence of OMMT in EPDM/silicone rubber nanocomposites improves the dielectric strength and volume resistivity.

It is known that porous polymer results in materials with good flexibility, lower electrical conductivity, and lower dielectric losses. To get the light-weighted MRNCs with a better MR effect, higher flexibility, and lower electrical and dielectric properties, two sets of fabricated MRNCs (porous and nonporous) based on silicon rubber matrix are selected to investigate the dielectric and electrical properties. The MRNCs samples are provided in five different weight percentage categories as $10 \%, 20 \%, 30 \%, 40 \%$, and $50 \%$ of nanosized CIPs (refer to [6]).

The contribution of current work is concerned with the experimental comparative study between porous and nonporous MRNCs through dielectric and electrical properties measurements and analysis to reach the design point of flexible magnetodielectric actuators.

\section{Experimental}

2.1. Dielectric Property Measurement. Disk shaped specimens of $0.8 \mathrm{~mm}$ thickness were made from film-shaped MRNCs samples. The specimens were prepared by using a sensitive electrical cutter. The surfaces of the test specimens were covered with copper thin films. The test samples were fixed between two electrodes and kept inside the sample holder. All the measurements were performed at a temperature of $27^{\circ} \mathrm{C}$ and at a relative humidity of $20 \%$. Besides that, the measurement environment is maintained constant so that they do not influence the results.

The capacitance and dielectric loss measurements in this study were performed by using a LCR HiTESTER (3532-50, Hioki, Japan). The measurements were carried out in the frequency range from $100 \mathrm{~Hz}$ to $0.1 \mathrm{MHz}$.

The dielectric constant $\left(\varepsilon^{\prime}\right)$ was calculated from the capacitance using the following equation [18-21]:

$$
\varepsilon^{\prime}=\frac{C t}{\varepsilon_{0} A},
$$

where $\varepsilon^{\prime}$ is the dielectric constant of the material, $\varepsilon_{0}$ is the permittivity of air $(8.85 \times 10-12 \mathrm{~F} / \mathrm{m}), C$ is the capacitance, $A$ is the area of cross-section of the sample, and $t$ is the thickness of the sample.

The loss tangent was estimated using the following equation:

$$
\tan \delta=\frac{\varepsilon^{\prime}}{\varepsilon^{\prime \prime}},
$$

where $\varepsilon^{\prime \prime}$ is referred to as loss factor of material.

2.2. Electrical Property Measurement. For the DC volume resistivity measurements, a Keithley electrometer (Model 8009) was used and the readings were obtained under an applied voltage of $250 \mathrm{~V}$ DC. The test was repeated three times for each MRNCs sample.

Through measuring the sample resistivity $(R)$ the volume resistivity can be determined as

$$
\rho=\frac{R \times A}{t},
$$

where $A$ is the cross-sectional area of the sample and $t$ represents the sample thickness between the two electrodes.

The electrical conductivity of sample is defined from the available volume resistivity values as

$$
\sigma=\frac{1}{\rho}=\frac{t}{R \times A} .
$$

\section{Results and Discussions}

3.1. Dielectric Property. The variations of dielectric constant and loss with respect to frequency for the porous and nonporous MRNCs samples at four different CIPs concentrations are shown in Figures 1 and 2, respectively.

It can be seen from the figures that the dielectric loss and constant of MRNCs samples decrease with increasing frequency and keep the frequency dependent behavior up to end of process.

The experimental measurements of dielectric properties of porous and nonporous MRNCs show that nonporous MRNCs have the higher dielectric loss and constants. Probably, this is due to the presence of a large number of charge 


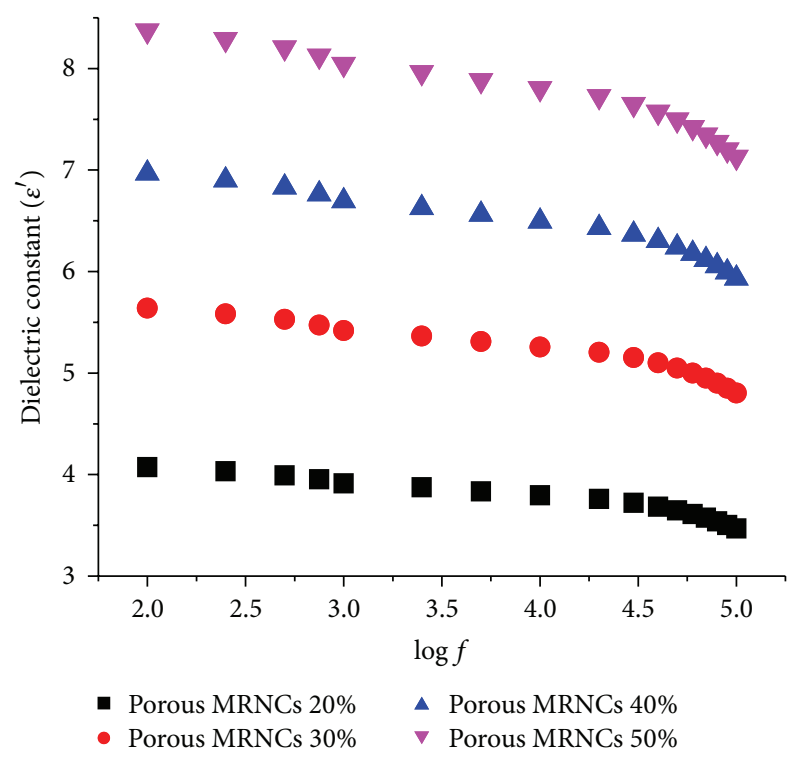

(a)

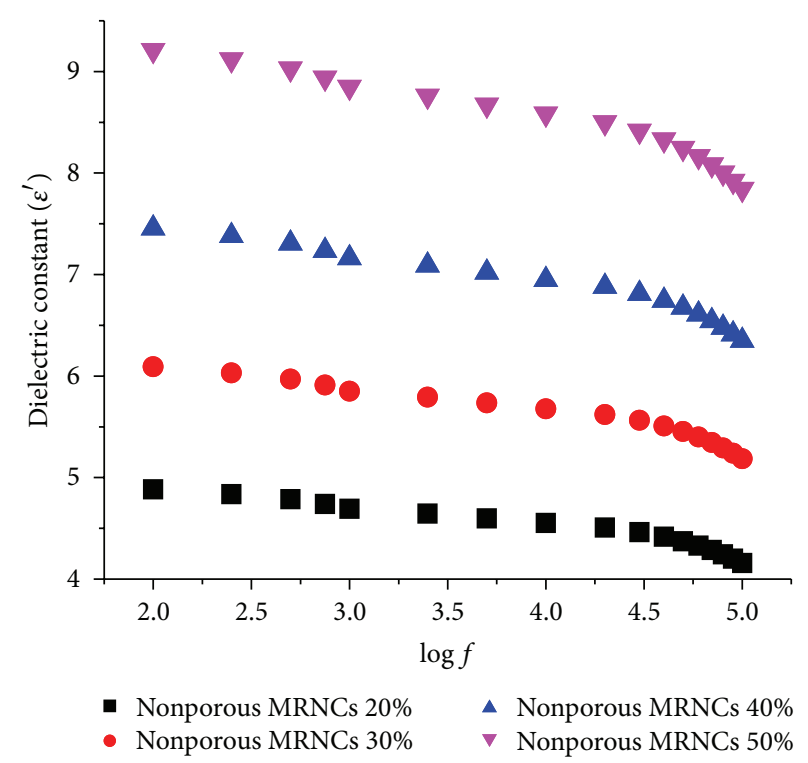

(b)

FIGURE 1: Dielectric constants for porous and nonporous MRNCs samples.

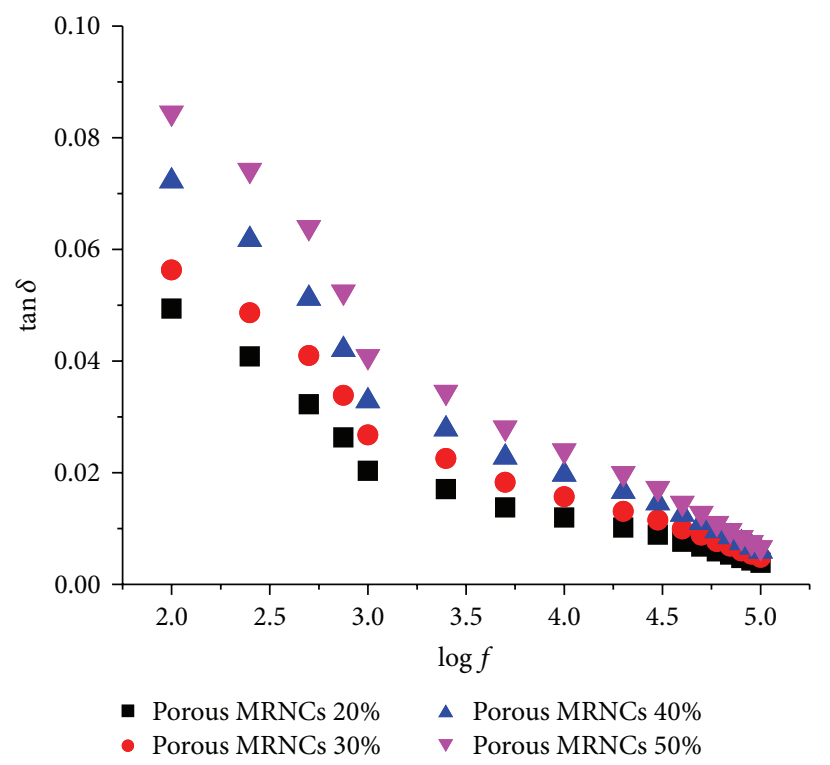

(a)

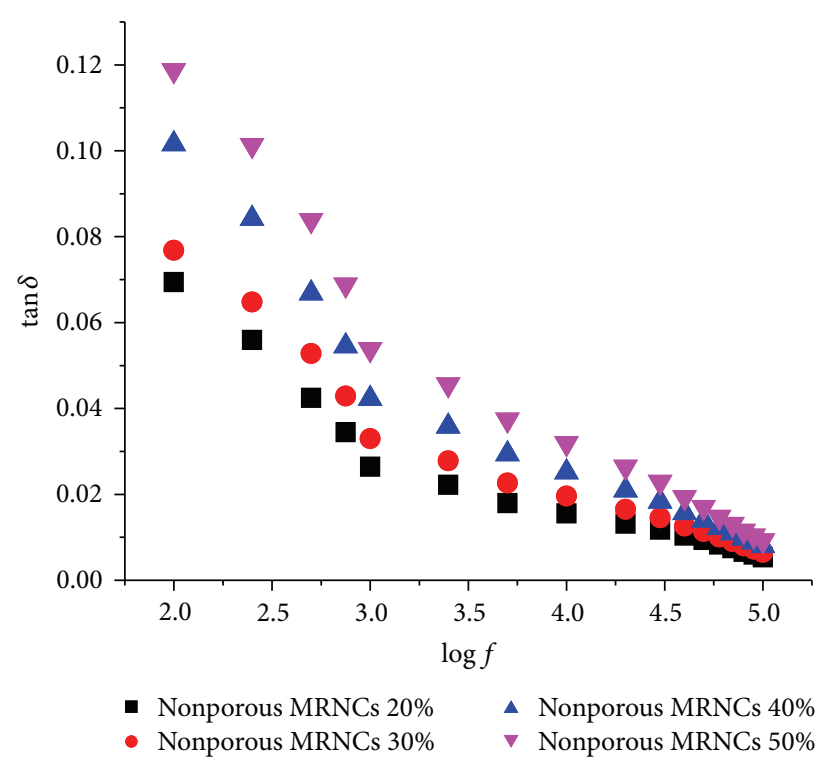

(b)

FIGURE 2: Dielectric loss for porous and nonporous MRNCs samples.

carriers in the bulk of nonporous MRNCs which are free to migrate under an applied electric. Besides that, the further addition of the CIPs into the nonporous silicone rubber increases the dielectric constant since the amount of interface increases in the composite.

The variations of MRNCs dielectric constants in Figure 1 show that there is an effect on the CIPs concentration percentages. In CIPs filled MRNCs sample, with increasing nanosized CIPs, the MRNCs dielectric constant increases. Experimentally, the dielectric properties of the MRNCs depend on the concentration level of CIPs and the interfacial properties of the MRNCs.

Of course, the main efforts are related to use of the current fabricated MRNCs for an actuator as well as gripper jaw. According to the experiments, the actuator performance is directly influenced by the stiffness and dielectric constant of silicone based nanocomposites as MRNCs. In terms of actuator strain induced by magnetic field, lower values of the dielectric constant are desirable. Accordingly, the dielectric constant should be minimized to give the desired 


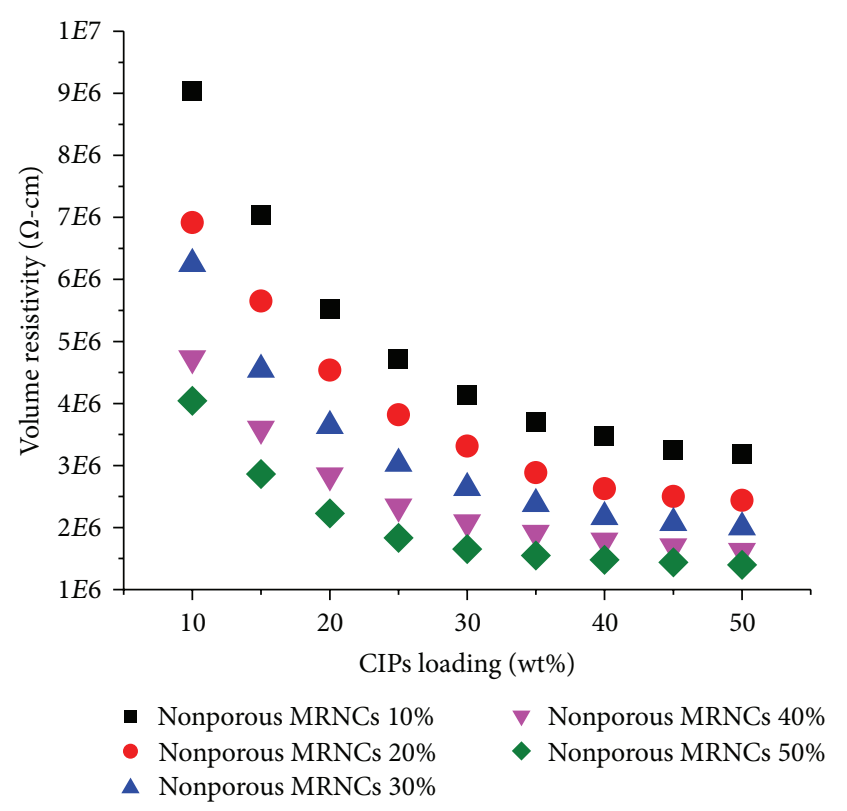

(a)

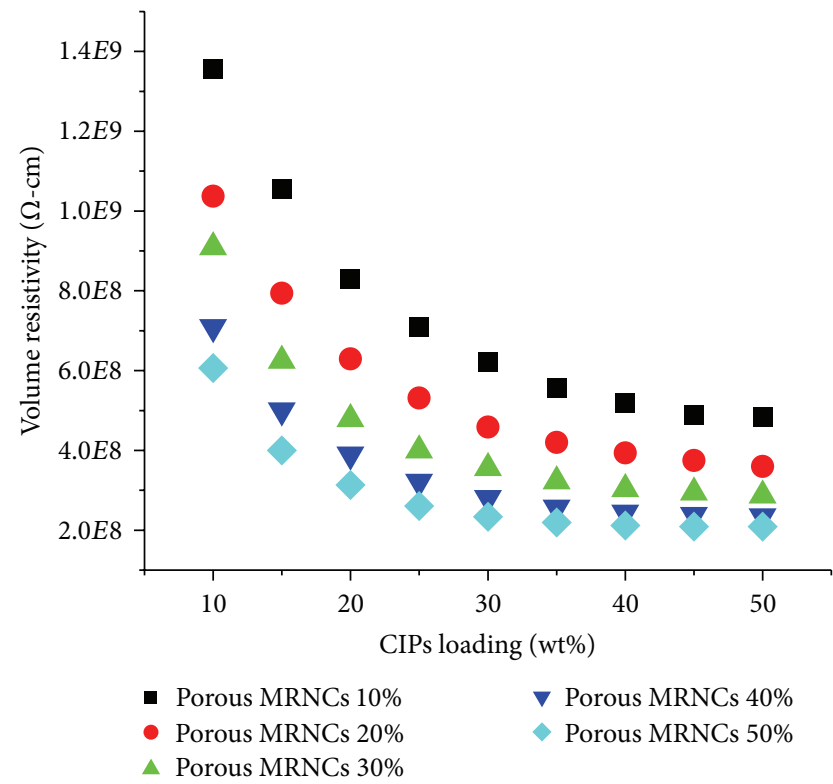

(b)

FIGURE 3: Volume resistivity profiles for nonporous and porous MRNCs sample.

performance of actuator. So, creating the pores will cause the decrement of dielectric constant of MRNCs.

As examined experimentally, the decreased dielectric constant provides increased strain of the MRNCs actuator (i.e., grippers).

It also should be mentioned that the morphology of the layered nanosized CIPs determines the dielectric constant of MRNCs. When CIPs are homogeneously dispersed (exfoliated) in a silicone rubber matrix [6], the dielectric constant is decreased.

The porous nanocomposites filled with nanosized CIPs exhibit the lowest dielectric constant value, whereas the nonporous MRNCs have the higher dielectric constant values, which can be explained by higher interfacial area in comparison to porous MRNCs based on silicone rubber.

3.2. Electrical Property. Figure 3 compares the variation of volume resistivity against nanosized CIPs loadings in porous and nonporous MRNCs samples.

From the figure, it was noticed that the volume resistivity decreased with increase of CIPs loadings due to the interaction of nanosized CIPs with silicone rubber matrix.

It can be concluded from Figure 3(b) that porous MRNCs show a higher value of resistivity as compared to the nonporous MRNCs filled with CIPs for the same filler loadings.

Basically, the motion of charge carriers contributing to the conductivity primarily occurs along the silicone rubber chains. A barrier to the charge transport in silicone rubbers (causing reduction in electrical conductivity) can occur due to defects, moisture content, interchain charge transport, interfaces related transport, and porosity.

Probably, the presence of a large number of interfaces, pores, and silicone rubber chain entanglements inhibits the
TABLE 1: Electrical conductivity of porous and nonporous MRNCs samples.

\begin{tabular}{lcc}
\hline \multirow{2}{*}{ Sample } & \multicolumn{2}{c}{ Conductivity $(\mathrm{S} / \mathrm{cm})$} \\
& Porous & Nonporous \\
\hline MRNCs 10\% & $1.96481 E-09$ & $2.95364 E-07$ \\
MRNCs 20\% & $2.60659 E-09$ & $3.77572 E-07$ \\
MRNCs 30\% & $3.33995 E-09$ & $4.60857 E-07$ \\
MRNCs 40\% & $4.1572 E-09$ & $5.76376 E-07$ \\
MRNCs 50\% & $4.90156 E-09$ & $7.03947 E-07$ \\
\hline
\end{tabular}

motion of charges in the nanocomposites, which in turn causes a reduction in the electrical conductivity (hence a lower tan delta value).

It also can be recorded that an increase in the weight percentage of nanosized CIPs loadings causes increase in conductivity as well (Table 1).

\section{Conclusions}

In this paper, the dielectric and electrical properties of two sets of isotropic MRNCs based on silicone rubber were compared and analyzed using experimental data. A series of desired dielectric and electrical property experiments were conducted to study the dielectric and electrical behaviors between nonporous and porous MRNCs samples. The porosity effects on dielectric and electrical properties of MRNCs samples are observed. The MRNCs with nanosized CIPs fillers display the advantageous dielectric behaviors at higher CIPs loadings. The dielectric constant and loss values in porous MRNCs are found to be lower than that of nonporous MRNCs. For both types of fabricated MRNCs, the dielectric 
constants and dielectric losses are decreasing with increase of frequency and related to the CIPs loadings. The results of comparison show that porosity plays an interesting role in the electrical characteristics changes of film-shaped MRNCs.

\section{Conflict of Interests}

The authors declare that there is no conflict of interests regarding the publication of this paper.

\section{References}

[1] R. Li and L. Z. Sun, "Dynamic mechanical behavior of magnetorheological nanocomposites filled with carbon nanotubes," Applied Physics Letters, vol. 99, no. 13, Article ID 131912, 2011.

[2] M.Zaborski and M. Masłowski, "Magnetorheological elastomer composites," Progress in Colloid and Polymer Science, vol. 138, pp. 21-26, 2011.

[3] A. Naimzad, Y. Hojjat, and M. Ghodsi, "Fabrication \& characterization of MR nanocomposites based on silicone rubber," in Proceedings of the 3rd International Conference on Composites: Characterization, Fabrication and Application (CCFA-3 '12), p. 121, Tehran, Iran, 2012.

[4] A. Naimzad, Y. Hojjat, and M. Ghodsi, "Study on MR Nanocomposites to develop a miniature gripper," in Proceedings of the International Conference on Actuator (Actuator '12), p. 616, Bermen, Germany, 2012.

[5] A. Naimzad, Y. Hojjat, and M. Ghodsi, "Attempts to design a miniature gripper using Magneto-Rheological Nanocomposites (MRNCs)," in Proceedings of the International Congress on Nanoscience \& Nanotechnology (ICNN'12), p. 488, Kashan, Iran, 2012.

[6] A. Naimzad, Y. Hojjat, and M. Ghodsi, "Comparative study on mechanical and magnetic properties of porous and nonporous film-shaped magnetorheological nanocomposites based on silicone rubber," International Journal of Innovative Science and Modern Engineering, vol. 2, no. 8, pp. 11-19, 2014.

[7] M. J. Jiang, Z. M. Dang, and H. P. Xu, "Enhanced electrical conductivity in chemically modified carbon nanotube/methylvinyl silicone rubber nanocomposite," European Polymer Journal, vol. 43, no. 12, pp. 4924-4930, 2007.

[8] Y. Duan, G. Li, L. Liu, and S. Liu, "Electromagnetic properties of carbonyl iron and their microwave absorbing characterization as filler in silicone rubber," Bulletin of Materials Science, vol. 33, no. 5, pp. 633-636, 2010.

[9] M. R. Kashani, S. Javadi, and N. Gharavi, "Dielectric properties of silicone rubber-titanium dioxide composites prepared by dielectrophoretic assembly of filler particles," Smart Materials and Structures, vol. 19, no. 3, Article ID 035019, 2010.

[10] O. Al-Hartomy, F. Al-Solamy, A. Al-Ghamdi, N. Dishovsky, V. Iliev, and F. EL-Tantawy, "Dielectric and microwave properties of silicone rubber/TiB ${ }_{2}$ nanocomposites," in Testing and Measuring, pp. 13-18, KGK, 2011.

[11] A. Katihabwa, W. Wang, Y. Jiang, X. Zhao, Y. Lu, and L. Zhang, "Multi-walled carbon nanotubes/silicone rubber nanocomposites prepared by high shear mechanical mixing," Journal of Reinforced Plastics and Composites, vol. 30, no. 12, pp. 1007-1014, 2011.

[12] H. Zou, S. Li, L. Zhang et al., "Determining factors for high performance silicone rubber microwave absorbing materials,"
Journal of Magnetism and Magnetic Materials, vol. 323, no. 12, pp. 1643-1651, 2011.

[13] I. Zivkovic and A. Murk, "Extraction of dielectric and magnetic properties of carbonyl iron powder composites at high frequencies," Journal of Applied Physics, vol. 111, Article ID 114104, 2012.

[14] B. G. Babu, D. E. Selvaraj, R. Srinivas et al., "Analysis of Relative Permittivity and tan delta characteristics of silicone rubber based nanocomposites," International Journal of Scientific Engineering and Technology, vol. 1, no. 5, pp. 201-206, 2012.

[15] I. Bica, "The influence of hydrostatic pressure and transverse magnetic field on the electric conductivity of the magnetorheological elastomers," Journal of Industrial and Engineering Chemistry, vol. 18, no. 1, pp. 483-486, 2012.

[16] H. Zhao, Y.-J. Xia, Z.-M. Dang, J.-W. Zha, and G.-H. Hu, "Composition dependence of dielectric properties, elastic modulus, and electroactivity in (carbon black- $\mathrm{BaTiO}_{3}$ )/silicone rubber nanocomposites," Journal of Applied Polymer Science, vol. 127, no. 6, pp. 4440-4445, 2013.

[17] V. Vijayalekshmi and S. S. M. Abdul Majeed, "Mechanical, thermal and electrical properties of EPDM/Silicone blend nanocomposites," International Journal of Engineering Research and Applications, vol. 3, no. 2, pp. 1177-1180, 2013.

[18] P. Barber, S. Balasubramanian, Y. Anguchamy et al., "Polymer composite and nanocomposite dielectric materials for pulse power energy storage," Materials, vol. 2, no. 4, pp. 1697-1733, 2009.

[19] D. C. Tiwari, V. Sen, and R. Sharma, "Temperature dependent studies of electric and dielectric properties of polythiophene based nano composite," Indian Journal of Pure and Applied Physics, vol. 50, no. 1, pp. 49-56, 2012.

[20] E. M. Abdul Jamal, P. A. Joy, P. Kurian, and M. R. Anantharaman, "Synthesis of nickel-rubber nanocomposites and evaluation of their dielectric properties," Materials Science and Engineering B: Solid-State Materials for Advanced Technology, vol. 156, pp. 24-31, 2009.

[21] S. M. Reda, "Electric and dielectric properties of $\mathrm{Fe}_{2} \mathrm{O}_{3} /$ silica nanocomposites," International Journal of Nano Science and Technology, vol. 1, no. 5, pp. 17-28, 2013. 

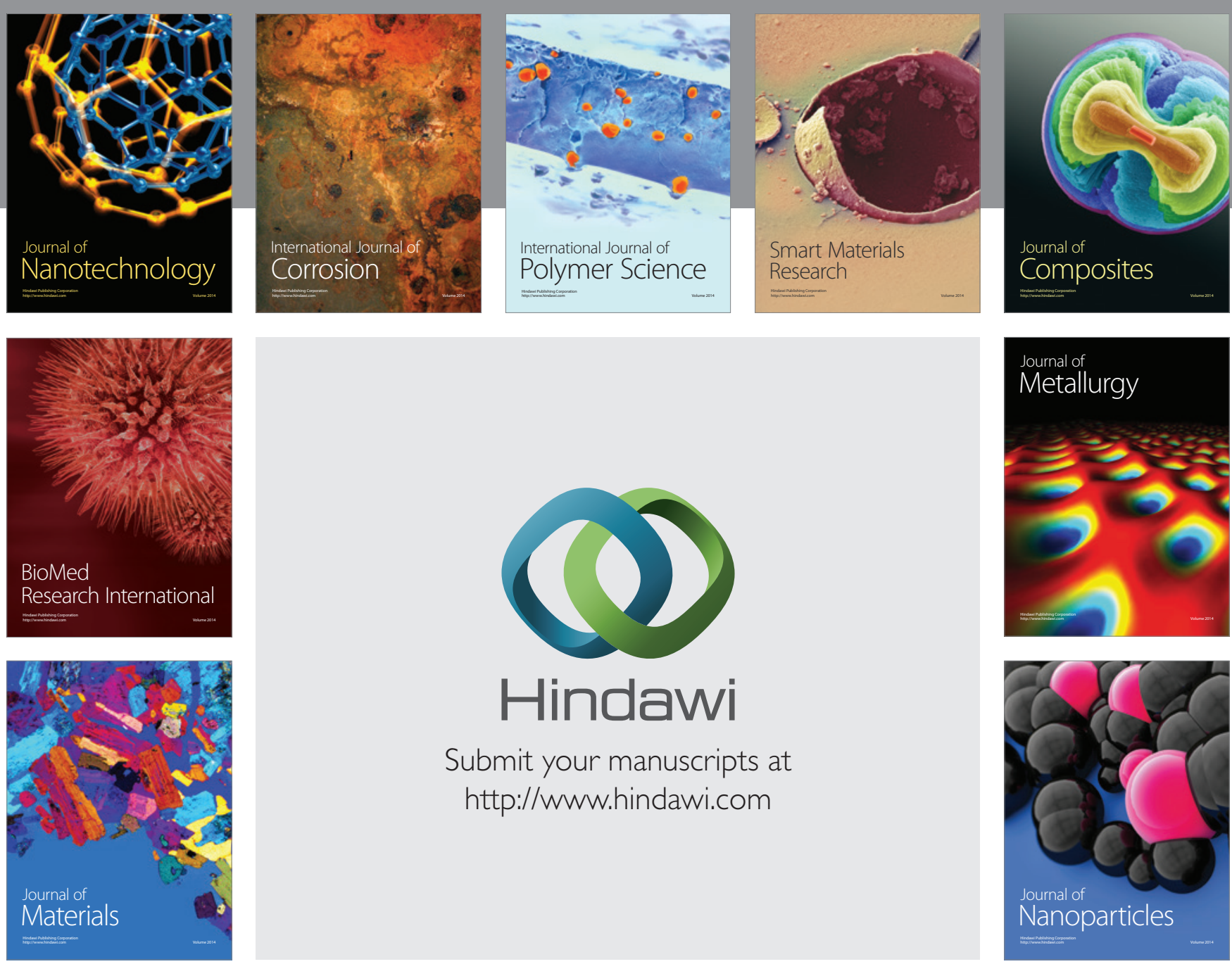

Submit your manuscripts at http://www.hindawi.com
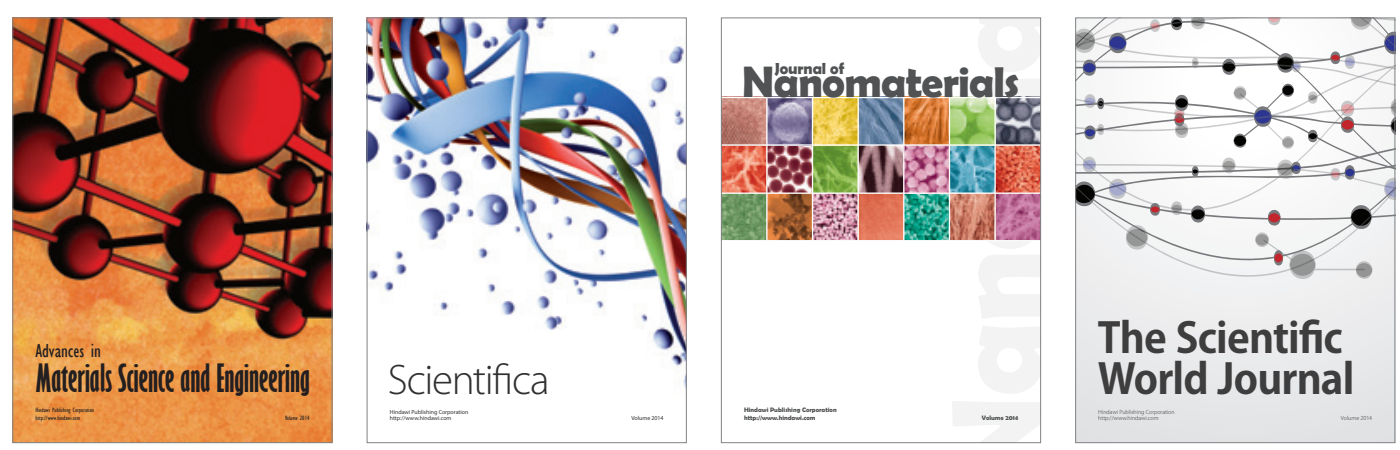

\section{The Scientific World Journal}
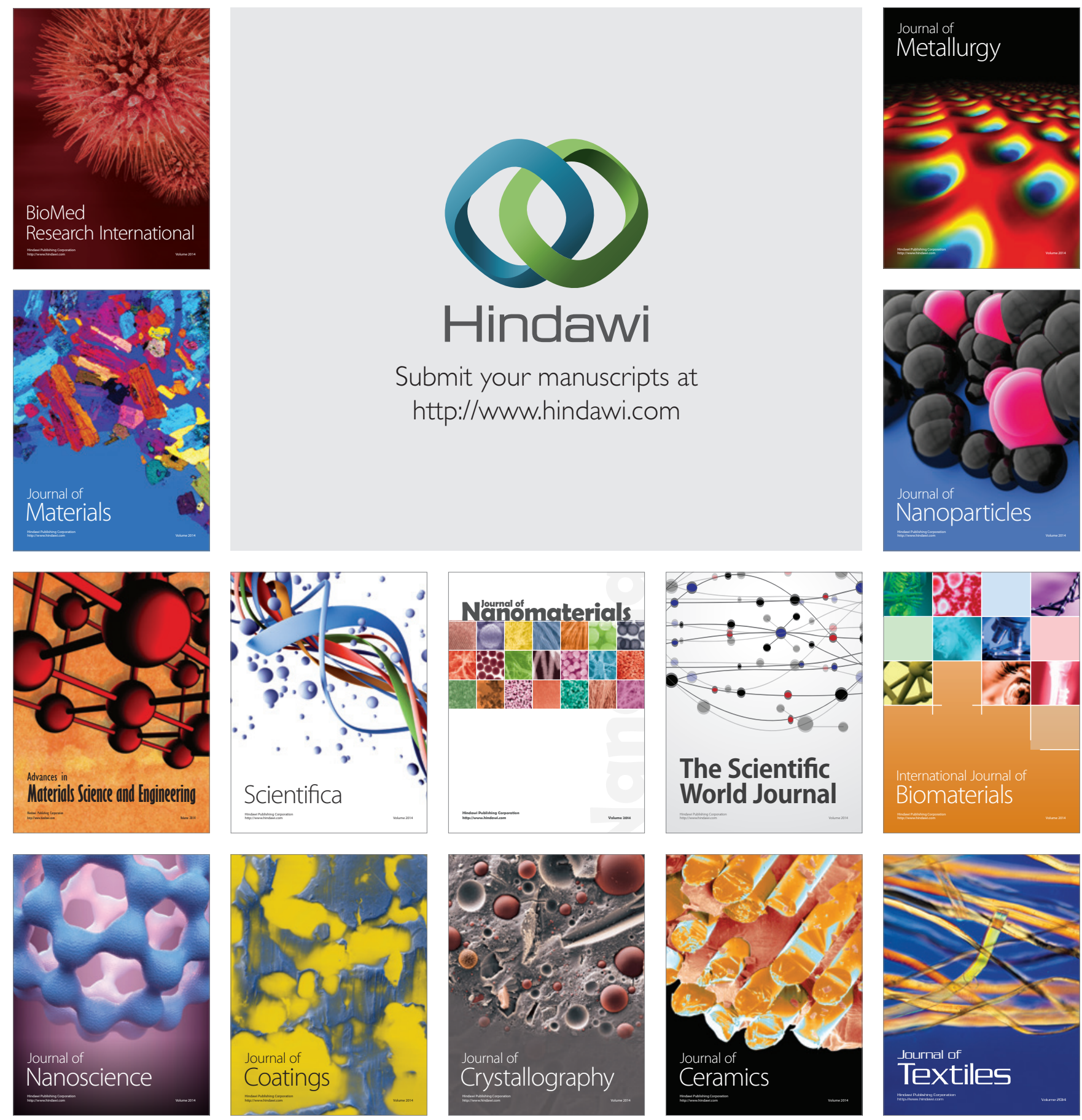